\title{
Caracterización comparativa de biopolímeros de almidón extraídos de cereales mediante dos técnicas diferentes
}

\section{Comparative characterization of starch biopolymers extracted from cereals using two different techniques}

\author{
RIVERA-ARREDONDO, Marisa †*”, RODRÍGUEZ-ÁNGELES, Mario Alberto“, MORALES-FÉLIX, \\ Verónica de Jesús"“ y GAYTÁN-RUELAS, Marina"”
}

'Procesos Alimentarios, Universidad Tecnológica del Suroeste de Guanajuato, Carretera Valle-Huanimaro K. 1.2, Valle de Santiago, Guanajuato, C.P: 38400, México.

\begin{abstract}
'Ingeniería de Plásticos, Universidad Politécnica de Juventino Rosas, calle Hidalgo 102, Comunidad de Valencia, Juventino Rosas, Guanajuato, 38253, México.
\end{abstract}

\author{
"Agricultura Sustentable y Protegida, Universidad Tecnológica del Suroeste de Guanajuato, Carretera Valle-Huanímaro \\ km 1.2, Valle de Santiago, Guanajuato, C.P.: 38400, México.
}

ID $1^{\text {er }}$ Autor: Marisa, Rivera-Arredondo

ID $1^{\text {er }}$ Coautor: Mario Alberto, Rodríguez-Ángeles

ID $2^{\text {do }}$ Coautor: Verónica de Jesús, Morales-Félix

ID $3^{\text {er }}$ Coautor: Mariana, Gaytán-Ruelas

DOI: $10.35429 / J S I .2020 .15 .4 .31 .36$

Recibido Julio 25, 2020; Aceptado Diciembre 30, 2020

\section{Resumen}

En el campo de investigación y consiguiente elaboración de biopolímeros ha ido creciendo en la última década, ya sea debido a endurecimiento de las legislaciones ambientales de cada país o por conciencia ecológica, en todo caso el término biopolímero es bastante amplio, siendo estos utilizados como materia prima en medicamentos, suplementos alimenticios y por supuesto en la elaboración de bioplásticos. Puesto que el tema de interés es la caracterización comparativa de biopolímeros usando almidón extraído de dos cereales diferentes avena y arroz mediante la técnica alcalina con tratamiento de $\mathrm{NaOH}$ y la técnica de molienda, presumiendo que el almidón extraído del cereal de Oryza sativa presenta características químicas, geométricas y mecánicas superiores en ambas técnicas utilizadas a diferencia de los polímeros elaborados con almidón de Avena sativa. Estos resultados sugieren que la calidad de almidón de cada cereal es relevante para la elaboración de biopolímeros [1]. Las propiedades comercialmente significativas del almidón, tales como su resistencia mecánica y flexibilidad, dependen de la resistencia y de carácter de la región cristalina, la cual depende de la relación de amilosa y amilopectina.

\begin{abstract}
In the field of research and consequent elaboration of biopolymers it has been growing in the last decade, either due to the hardening of the environmental legislation of each country or due to ecological awareness, in any case the term biopolymer is quite broad, these being used as material premium in medicines, food supplements and of course in the production of bioplastics. Since the topic of interest is the comparative characterization of biopolymers using starch extracted from two different cereals, oats and rice, using the alkaline technique with $\mathrm{NaOH}$ treatment and the milling technique, assuming that the starch extracted from the Oryza sativa cereal presents chemical characteristics, geometric and mechanical superior in both techniques used unlike the polymers made with Avena sativa starch. These results suggest that the starch quality of each cereal is relevant for the production of biopolymers [1]. The commercially significant properties of starch, such as its mechanical strength and flexibility, depend on the strength and character of the crystalline region, which depends on the ratio of amylose and amylopectin.
\end{abstract}

Citación: RIVERA-ARREDONDO, Marisa, RODRÍGUEZ-ÁNGELES, Mario Alberto, MORALES-FÉLIX, Verónica de Jesús y GAYTÁN-RUELAS, Marina. Caracterización comparativa de biopolímeros de almidón extraídos de cereales mediante dos técnicas diferentes. Revista de Innovación Sistemática. 2020. 4-15:31-36.

$\dagger$ Investigador contribuyendo como primer autor. 


\section{Introducción}

\section{Revisión literaria}

Propiedades estructurales del almidón natural.

Los granos de almidón están formados por macromoléculas organizadas en capas. Dos estructuras poliméricas diferentes componen los almidones: la amilosa y la amilopectina. Cerca del $20 \%$ de la mayoría de los almidones es amilosa y el $80 \%$ amilopectina. Las moléculas de amilosa, situadas en las capas interiores, están compuestas de aproximadamente 200 a 20,000 moléculas de glucosa unidas por enlaces glucosídicos $\alpha-1,4$ (Figura 1) en cadenas no ramificadas o enrolladas en forma de hélice. Muchas moléculas de amilosa tienen algunas ramificaciones $\alpha-\mathrm{D}-(1,6)$, aproximadamente entre 0.3 a $0.5 \%$ del total de los enlaces. Estas generalmente, no son ni muy largas ni muy cortas y están separadas por grandes distancias permitiendo a las moléculas actuar, esencialmente con un polímero lineal, formando películas y fibras fuertes, y retrogradado fácilmente. Como consecuencia de la formación de cadenas en forma de hélice las fibras y películas de amilosa son más elásticas que las de celulosa. La amilosa es soluble en agua caliente lo cual se debe a la formación de una suspensión coloidal. [2]

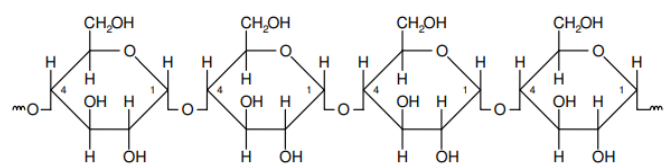

Figura 1 Segmentos de una molécula de amilosa Fuente: [5]

La estructura de la amilopectina, situada en las capas exteriores, es diferente a la de la amilosa. Las moléculas de la amilopectina contienen enlaces glicosidicos $\alpha-1,4$ y $\alpha-1,6$, como se observa en la figura 2 . Los enlaces glicosidicos unen las moléculas de glucosa en la cadena principal de amilopectina. Con frecuencia se encuentran ramificaciones de la cadena principal, las cuales se deben a los enlaces glicosidicos $\alpha-1,6$ con otras moléculas de glucosa. Los puntos de enlace de las ramificaciones constituyen entre el 4 y $5 \%$ del total de los enlaces. [6]
Las moléculas de amilopectina son significativamente más grandes que las moléculas de amilosa; algunas contienen entre 10,000 y 20 millones de unidades de glucosa [7]. El peso molecular de la amilosa está entre 0.1 y un millón de g/mol. Y el de la amilopectina está entre (10,000 a 1,000 millones) $\mathrm{g} / \mathrm{mol}$ [5]. Una de las propiedades más importantes del almidón natural es su semicristalinidad donde la amilopectina es el componente dominante para la cristalización en la mayoría de los almidones. La parte amorfa está formada por regiones ramificadas de amilopectina y amilosa. Las propiedades comercialmente significativas del almidón, tales como su resistencia mecánica y flexibilidad, dependen de la resistencia y de carácter de la región cristalina, la cual depende de la relación de amilosa y amilopectina y por lo tanto del tipo de planta, de la distribución del peso molecular, del grado de ramificación y del proceso de conformación de cada componente del polímero. [8]

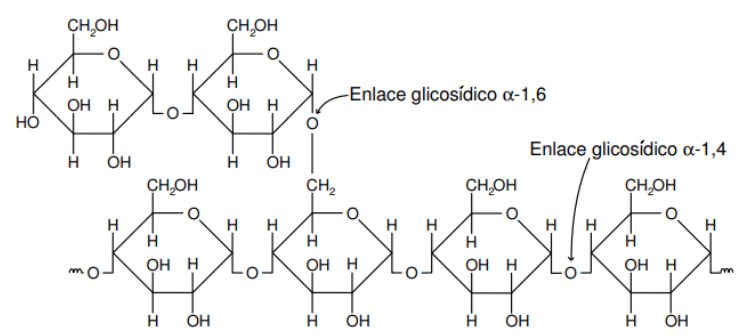

Figura 2 Segmento de una molécula de amilopectina Fuente: [5]

Gelatinización.

Se define como la perdida de cristalinidad de los granos de almidón en presencia de calor y altas cantidades de agua con muy poca o ninguna despolimerización. Los granos de almidón son insolubles en agua y en solventes orgánicos. En suspensión acuosa los granos se hinchan por la acción del calor, tienden a perder las propiedades que le confiere su estructura semicristalina y a una temperatura crítica forman un gel. [8] 
Durante la gelatinización el agua penetra inicialmente en las regiones amorfas iniciando el hinchamiento, lo cual se aprecia por la disminución en la birrefringencia. Luego el agua desaloja las cadenas de almidón desde la superficie de los cristales a medida que la temperatura; la movilidad térmica de las moléculas y la solvatación producida por las fuerzas de hinchamiento provocan una disminución de la cristalinidad por el desenrollado de las dobles hélices, hasta que la estructura granular es fragmentada casi completamente obteniéndose un sólido-gel. La principal diferencia entre la preparación de geles, comidas, películas o materiales procesados de almidón termoplástico (TPS) es la cantidad de agua o plastificante durante la gelatinización o fusión de los gránulos de almidón. Para la obtención del almidón termoplástico, el almidón se funde con la ayuda de una cantidad relativamente baja de agua durante el proceso de extrusión, moldeo por presión o moldeo por inyección, por donde la cantidad de agua está por debajo del $20 \%$ en la mayoría de los casos. Parte del agua generalmente se reemplaza por pequeñas cantidades de glicerina. Las diferencias en el contenido de agua y glicerina y las condiciones del procesamiento tales como: la velocidad de Obtención de un polímero biodegradable a partir de almidón de maíz cizalladura y temperatura producen diferencias en la formación de la red de almidón y en la morfología del material producido. [9]

El almidón, como materia prima para la elaboración de un bioplástico

Para convertir un almidón seco en un material bioplástico es necesario romper y fundir la estructura granular semicristalina del mismo [10]. El almidón sin los aditivos adecuados (plastificantes) no tiene las propiedades necesarias para trabajar como termoplástico, Los plastificantes incrementan la flexibilidad del almidón debido a su habilidad para reducir la interacción de los enlaces de hidrógeno, además de aumentar el espacio molecular [12].
Se pueden distinguir dos tipos de ordenamiento en el almidón termoplástico después de su procesamiento: la cristalinidad residual clasificada en las formas tipo A, B y C causadas por la fusión incompleta durante la plastificación y la cristalinidad inducida durante el procesamiento, de acuerdo con los arreglos generados en las cadenas poliméricas según [11] el tipo A es común en almidones de cereales, el $\mathrm{B}$ en tubérculos y el $\mathrm{C}$ en ciertas raíces y semillas. La cantidad de cristalinidad residual está relacionada con la temperatura y el esfuerzo de corte aplicado durante el procesamiento; de igual manera la composición de la mezcla de alimentación también influye indirectamente en esta cantidad de cristalinidad remanente. Dependiendo de algunas condiciones de procesamiento y almacenamiento como la temperatura y la humedad, el almidón amorfo sufre cambios estructurales después del enfriamiento, basados en: recristalización de la amilosa y la amilopectina en diferentes estructuras cristalinas, separación de fase y reorientación del polímero. Las interacciones moleculares (principalmente puentes de hidrógeno entre las cadenas de almidón) que ocurren después del enfriamiento son llamadas retrogradación [10]. Esta retrogradación hace referencia igualmente a los cambios que tienen lugar en el almidón gelatinizado desde un estado amorfo inicial a uno cristalino más ordenado. Ocurre porque los geles de almidón no son termodinámicamente estables. De acuerdo a las cadenas de amilopectina son responsables por los fenómenos de retrogradación que se generan a largo plazo, mientras que la amilosa se relaciona con los cambios a tiempos más cortos. [10]

\section{Materiales y métodos}

\section{Extracción de almidón}

El método de extracción de almidón se realiza por el método utilizado por Yamamoto, 1973 y algunas modificaciones. Donde se usó dos metodologías diferentes en cada cereal [5]. 
En la primera metodología se usaron 100 gramos de Oryza sativa y 100 gramos de Avena sativa se sometieron a una serie de lavados, primeramente, con $500 \mathrm{ml}$ de agua destilada cada uno, posteriormente se colocaron $500 \mathrm{ml}$ de $\mathrm{NaOH}$ al $0.1 \mathrm{~N}$, continuando con agua destilada, y finalmente una neutralización con $\mathrm{HCl}$ al 0.5 M por último un lavado con agua destilada y se centrifugó a 3000 rpm durante 20 minutos y un secado a $60{ }^{\circ} \mathrm{C}$ durante 48 horas. El proceso concluyó con una molienda y un tamizado. Cabe mencionar que entre cada lavado se mantuvo un reposo de 24 horas a $6{ }^{\circ} \mathrm{C}$. La segunda metodología se usó las mismas cantidades $100 \mathrm{~g}$ de cada cereal y consistió en dejar reposar a ambos cereales con agua durante 24 horas. Posteriormente se realizó una molienda simple, se centrifugó a 3000 rpm durante 20 minutos. Se les retiró el exceso de agua y el residuo se llevó a secar en cápsulas de porcelana a una estufa cuyos parámetros fueron $60^{\circ} \mathrm{C}$ durante 48 horas. El proceso concluyó con una molienda y un tamizado. [13]

\section{Elaboración de los biopolímeros.}

Para la elaboración de los biopolímeros mediante una hidrolisis acida se llevó a cabo mediante la metodología de Rosales 2016 y adecuaciones. Donde se realizó la misma metodología la variante fue el almidón de cereales. Tabla 1, posteriormente se realizó la mezcla de cada uno de los componentes y se sometieron a un calentamiento sobre una placa térmica a $80{ }^{\circ} \mathrm{C} 15$ minutos, se vertió en papel encerado para después ser extendido mediante placas metálicas y llevado a secar durante 48 horas a una temperatura de $30^{\circ} \mathrm{C}$. Esto se realizó con cada muestra de almidón por triplicado. [14]

\begin{tabular}{|c|c|c|c|c|c|}
\hline $\begin{array}{c}\text { Cereal y } \\
\text { formas } \\
\text { de } \\
\text { extracción } \\
\text { del } \\
\text { almidón }\end{array}$ & $\begin{array}{c}\text { Cantidad } \\
\text { (g) }\end{array}$ & $\begin{array}{c}\text { Agua } \\
\text { destilada } \\
(\mathrm{ml})\end{array}$ & $\begin{array}{c}\text { Acido } \\
\text { cítrico } \\
\text { (g) }\end{array}$ & $\begin{array}{c}\text { Glicerina } \\
(\mathrm{ml})\end{array}$ & $\begin{array}{c}\text { Nomenclatura } \\
\text { del } \\
\text { tratamiento }\end{array}$ \\
\hline $\begin{array}{l}\text { Oryza } \\
\text { sativa } \\
\mathrm{NaOH}\end{array}$ & 5 & 15 & 2.5 & 1.5 & T2SO \\
\hline $\begin{array}{l}\text { Oryza } \\
\text { sativa } \\
\text { Molienda } \\
\text { simple }\end{array}$ & 5 & 15 & 2.5 & 1.5 & T1MO \\
\hline $\begin{array}{l}\text { Avena } \\
\text { sativa } \\
\mathrm{NaOH}\end{array}$ & 5 & 15 & 2.5 & 1.5 & T2SA \\
\hline $\begin{array}{l}\text { Avena } \\
\text { sativa } \\
\text { Molienda } \\
\text { simple }\end{array}$ & 5 & 15 & 2.5 & 1.5 & T1MA \\
\hline
\end{tabular}

Tabla 1 Elaboración de biopolímeros Fuente: Elaboración propia

\section{Análisis químico y mecánico}

La determinación de humedad se llevó a cabo por el método descrito por la NMX-F-0831986. Así mismo, la determinación de cenizas se llevó a cabo por los lineamientos descritos por la NMX-F-066-S-1978. La determinación de resistencia fue realizada en un analizador de textura, 0 a $1500 \mathrm{~g} / 0.20 \mathrm{~g}$, Brookfield, CT31500 , donde se colocó un biopolímero con dimensiones de $15 \mathrm{~cm}$ de largo y 15 de alto. La fuerza tensil se obtuvo dividiendo la fuerza máxima antes de que se rompiera la película entre el grosor transversal de esta. Se reporta la fuerza de corte en $\mathrm{N}$.

\section{Análisis geométrico}

La determinación del grosor (mm) mediante un Calibre Vernier de $0-150 \mathrm{~mm}$ constó en colocar un fragmento de muestra es las puntas para medidas externas y posteriormente se tomó la lectura en el marcador digital.

\section{Resultados}

Elaboración de biopolímeros

En la figura 3 muestra los biopolímeros de Oryza sativa con la técnica de extracción de almidón alcalina (T2SO) y molienda simple (TIMO) y con características prometedoras en cuanto a su fuerza de rompimiento y calidad de almidón, donde se puede observar una de las propiedades más importantes de los almidones naturales, que es su semicristalinidad donde la amilopectina es el componente dominante para su cristalización que se le atribuye a una resistencia mecánica favorable debido a la relación de amilosa y amilopectina con un valor de $(36.41 \pm 2.2$ y 62.75 \pm 2.2$)$. [15] De acuerdo con su temperatura de gelatinización del almidón varían de $70 \mathrm{a}>75^{\circ} \mathrm{C}$. [16]

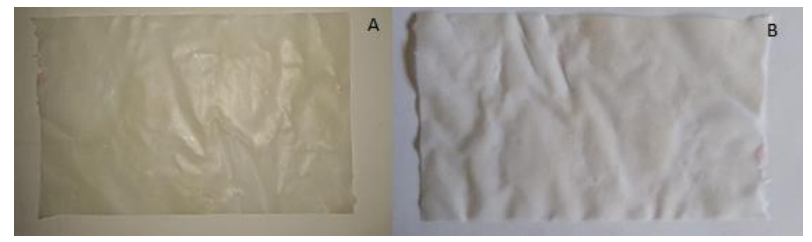

Figura 3 Biopolímeros de Oryza sativa. A biopolímero mediante la extracción de almidón en solución alcalina (T2SO). B biopolímero mediante extracción de almidón por molienda simple (T1MO)

Fuente: Elaboración propia 
En la figura 4 muestra los biopolímeros de Avena satina con sus dos técnicas de extracción de almidón claramente se puede observar que hay una menor resistencia a su fuerza de rompimiento. El contenido de amilosa es relativamente menor comparado con el de otros cereales [17]. Los almidones de avena presentaron las temperaturas de gelatinización menores, entre 56.8 y $59.7^{\circ} \mathrm{C}$, usando la misma temperatura para la elaboración de los biopolímeros perdieron mayor humedad generando poca resistencia mecánica.

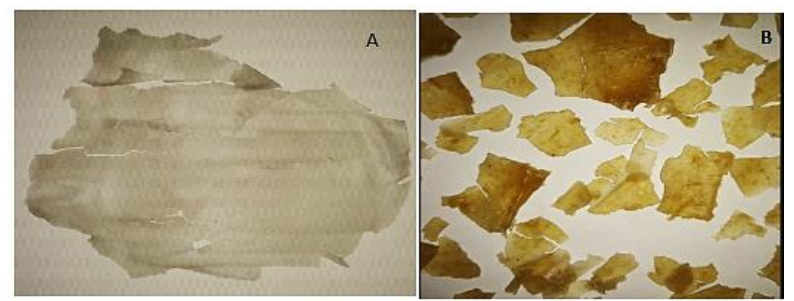

Figura 4 Biopolímero de Avena satina. A biopolímero extraído mediante solución alcalina (T2SA). B biopolímero extraído mediante molienda simple (T1MA) Fuente: Elaboración propia

\section{Análisis químico y mecánico}

La humedad relativa con menor porcentaje la presento el polímero T1MA y siguiendo el T2SA ambos son el mismo almidón técnica diferente de extracción. Lo que indica que la Avena Sativa presenta una menor absorción por su región semicristalina con menor relación de amilosa y amilopectina, generando una perdida más rápida de agua y generando menor resistencia mecánica. Tabla 2. Confirmando con su porcentaje de cenizas menores en los almidones de Avena sativa.

\begin{tabular}{|r|c|r|r|}
\hline \multicolumn{1}{|c|}{ T1MA } & T2SA & T1MO & T2SO \\
\hline $5.08 \%$ & $7.55 \%$ & $9.30 \%$ & $9.32 \%$ \\
\hline \pm 0.15 & \pm 0.16 & \pm 0.05 & \pm 0.03 \\
\hline
\end{tabular}

Tabla 2 Valores de humedad en los tratamientos realizados

Fuente: Elaboración propia

\begin{tabular}{|r|r|r|r|}
\hline \multicolumn{1}{|c|}{ T1MA } & \multicolumn{1}{c|}{ T2SA } & T1MO & T2SO \\
\hline $1.77 \%$ & $0.66 \%$ & $2.11 \%$ & $1.33 \%$ \\
\hline \pm 0.33 & \pm 0.2 & \pm 0.33 & \pm 0.3 \\
\hline
\end{tabular}

Tabla 3 Valores de cenizas en los tratamientos realizados Fuente: Elaboración propia
Determinación de la fuerza de rompimiento

La fuerza de corte se obtuvo dividiendo la fuerza máxima antes de que se rompiera la película entre el grosor transversal de esta. Se reporta la fuerza de corte en $\mathrm{N}$.

Únicamente se determinó con T1MO y T2SO por la apariencia de sus superficies ya que los tratamientos T1MA y T2SA presentaron agrietamientos en su toda su superficie. Confirmando que se atribuye en la calidad del almidón de cereales. Tabla 4.

\begin{tabular}{|c|r|r|r|}
\hline $\begin{array}{c}\text { T1MO } \\
\text { N }\end{array}$ & \multicolumn{1}{c}{ T2SO } & \multicolumn{1}{c|}{ T1MA } & T2SA \\
$\mathbf{N}$ & \multicolumn{1}{c|}{$\mathbf{N}$} & \multicolumn{1}{c|}{$\mathbf{N}$} \\
\hline 2.70 & 2.90 & 0 & 0 \\
\hline \pm 0.02 & \pm 0.05 & 0 & 0 \\
\hline
\end{tabular}

Tabla 4 Valores de la determinación de la fuerza de rompimiento

Fuente: Elaboración propia

\section{Análisis geométricos}

Por último, se realizó la determinación de grosor, mostrando los tratamientos, así como sus deviaciones estándar no hay diferencia significativa con respecto al grosor que pueda atribuir a la resistencia o bien a su contenido de humedad y cenizas. Tabla 5.

\begin{tabular}{|c|c|c|c|}
\hline $\begin{array}{c}\text { T1MA } \\
\text { mm }\end{array}$ & $\begin{array}{c}\text { T2SA } \\
\text { mm }\end{array}$ & $\begin{array}{l}\text { T1M0 } \\
\text { mm }\end{array}$ & $\begin{array}{c}\text { T2SO } \\
\text { mm }\end{array}$ \\
\hline 0.61 & 0.65 & 0.61 & 0.68 \\
\hline \pm 0.14 & \pm 0.02 & \pm 0.06 & \pm 0.13 \\
\hline
\end{tabular}

Tabla 5 Grosor de los tratamientos realizados Fuente: Elaboración propia

\section{Conclusiones}

La proporcion de amilosa y amilopectina juegan un papel importante en las propiedades de almidones termoplasticos. La temperatura de gelatinización es aquella en la cual los granos de almidón empiezan absorber agua e hincharse en forma irreversible en agua caliente, está asociada con el contenido de amilosa. Lo que se puede distinguir es dos tipos de ordenamiento en el almidón termoplástico después de su procesamiento: la cristalinidad residual clasificada en las formas tipo A, B y C causadas por la fusión incompleta durante la plastificación y la cristalinidad inducida durante el procesamiento, de acuerdo con los arreglos generados en las cadenas poliméricas según. [11]. 
El tipo A es común en almidones de cereales, el B en tubérculos y el $\mathrm{C}$ en ciertas raíces. Lo que indica que a pesar de que el almidón pertenece a la misma clasificación de cristalinidad residual. La relación de amilosa y amilopectina es diferente en cada tipo de cereal, de acuerdo a los análisis realizados se obtuvo que el almidón extraído por ambas técnicas de extracción de Oryza Sativa presentan mejores propiedades de biopolímeros lo que atribuye que lo importante es el almidón y no hay diferencia significativa con respecto al método de extracción dela almidón.

\section{Referencias}

[1] James E. Mark et al. High performance biodegradable materials from oriented starch derivates. U. S. patent 6,218,532 Cincinnati, USA, 2001.

[2] Mario Demicheli. Biodegradable plastics from renewable sources http://www.jrc.es/pages/iptsreport/vol10/en glish/Env1E106.htm, 2000.

[3] Biocorp@. http://www.BiocorpUSA.com, 2002

[4] S. Mali et al. Microestructural characterization of Yam starch films, Carbohydrate Polymers, 50, 379-386 (2002).

[5] P. Matzinos et al. Processing and characterization of LDPE/starch products, Journal of Applied Polymer Science, 79, 2548-2557 (2000).

[6] R. L. Whistler y J. N. BeMiller. Carbohydrate chemistry for food scientists. St Paul: Eagan Press, 1997.

[7] Drew H. Wolfe. Qu'imica General Org'anica. McGraw-Hill segunda edici'on, 432- 433 (1996).

[8] H. G. Fritz. Study of production of thermoplastics and fibers based mainly on biological material. European commission. Stuttgart German, 392 (1994). Universidad EAFIT 27| Obtenci' on y caracterizaci'on de un pol'imero biodegradable a partir del almid'on de yuca
[9] J. Van Soest et al. Mechanical properties of thermoplastic waxy maize starch, Journal of Applied Polymer Science, 6, 1927-1937 (1996).

[10] Thiré, M.S.M.R., R.A. Simâo. y Andrade, T.C; Investigation of the surface morphology of plasticized cornstarch films, Acta Micro.: 12 (1), 175-179 (2003b)

[11] Van Soest, J.J.G. y otros tres autores; Changes in the mechanical properties of thermoplastic potato starch in relation with changes in B-type crystallinity, Carbohydr. Polym: 29 (3), 225-232 (1996b).

[12] Mali, S. y otros tres autores; Water sorption and mechanical properties of cassava starch films and their relation to plasticizing effect, Carbohydr. Polym: 60 (3), 283-289 (2005a).

[13] Yamamoto, K., Sawada, S., Onogaki, T. Properties of rice starch prepared byalkali method with various conditions. Denpun Kagaku (1973) 20, 99-104.

[14] Rosales. Obtención de biopolímeros plásticos a partir del almidón de malanga por el método de polimerización por condensación (2016)

[15] Landires, D.., Márquez G, Facultad de Ingeniería en Mecánica y Ciencias de la producción Escuela superior politécnica de litoral 09-01-5863 2013

[16] Manriquez, C., Cuevas F. Evaluacion de la calidad culinaria y molienda del arroz Cali, Colombia Pags 22-25

[17] Welch, R. W., \& McConnell, J. M. (2001). Avena. En D. A. V. Dendy \& B. J. Dobraszczyk (Eds.), Cereales y Productos Derivados: Química y Tecnología (pp. 457, 460, 461). Zaragoza, España: Editorial Acribia. 